\title{
The Hel abundance in Solar filaments
}

\author{
F. Chiuderi Drago
}

Department of Astronomy and Space Science, University of Florence, Italy

Received 29 April 2005 / Accepted 29 July 2005

\section{ABSTRACT}

Three filaments observed with the CDS instrument on the SOHO satellite are analysed to determine the HeI/HI ratio. The HI and Hel boundfree absorptions are the major processes responsible for the lower intensity of transition region (TR) lines observed above filaments. One of the filaments was also observed by SUMER at $\lambda>912 \AA$, thus supplying the unabsorbed background intensity. The HI and Hel column densities are derived from several TR lines using a least squares method applied to two different models. The resulting HeI/HI ratio is independent of the model, while the column densities are different by about a factor of two. This difference enables us to discriminate between the two models by comparing the resulting value of the optical depth at the Lyman continuum limit, $\tau_{912}$, with previous observations and models.

Key words. Sun: filaments - Sun: UV radiation

\section{Introduction}

Since the launch of the Solar and Heliospheric Observatory (SoHO), several observations of disk filaments have been preformed. The main purpose for observations of filaments and prominences in UV and EUV lines was, at the beginning, the analysis of the thin prominence-corona transition region (PCTR). Our knowledge of the quiet sun transition region (TR) comes in fact from the analysis of UV and EUV lines formed in the temperature range $2 \times 10^{4}-1 \times 10^{6} \mathrm{~K}$ and of the microwaves radiation.

It became clear, by analysing a filament observed by the Solar Ultraviolet Measurements of Emitted Radiation (SUMER) and the Coronal Diagnostic Spectrometer (CDS), that the PCTR emission above the filament is negligible and that the lower TR line intensity observed at the filament site (only at $\lambda<912 \AA$ ) was due to the Lyman continuum (Ly-C) absorption of the radiation coming from the underlying quiet sun TR within the cool prominence body (Chiuderi Drago et al. 2001, Paper I). If the quiet sun TR emission under the filament (the background) is lower than the average, the PCTR emission could be not negligible.

Heinzel et al. (2001) have shown that the Ly-C optical depth of a given neutral hydrogen column density is indeed much larger than the corresponding $\mathrm{H}_{\alpha}$ optical depth, thus making the filaments more extended in UV lines than in $\mathrm{H}_{\alpha}$.

The possibility that HeI (and HeII) absorption may also affect the opacity of lines with $\lambda<504 \AA(\lambda<228 \AA)$ has been recently considered by Anzer \& Heinzel (2005), who computed the continuum opacity for three iron lines at $\lambda=171 \AA$, $195 \AA$ and $284 \AA$, assuming two different values of the He first ionization degree, $j_{1}=N_{\mathrm{HeII}} / N_{\mathrm{He}}$. however the results were almost insensitive to this quantity.
Del Zanna et al. (2004, Paper II) have checked, on three analysed filaments, how the $\mathrm{HeI} / \mathrm{HI}$ abundance can influence the observed line intensities. However, since no rigorous statistical procedure was applied, these results cannot be considered a good quantitative estimate of the above ratio.

In the present paper we will reconsider three out of the four filaments previously analyzed to quantitatively determine the $\mathrm{HeI} / \mathrm{HI}$ ratio in these features.

In the next section the $\mathrm{HI}$ and HeI column densities will be derived using a least squares method applied to the two prominence models mentioned in Paper II: model A which assumes an isothermal cool gas in the prominence with a TR all around it and model B in which the prominence is made up of a number $N$ of cool threads embedded in the hot coronal plasma with a thin tube-like TR around each of them (Chiuderi Drago et al. 1992, and references therein). A third parameter will be determined from the least squares procedure, namely the background emission in model A, for different values of the PCTR emission and the the total PCTR emission in model B, where the background emission is neglected.

In Sect. 3 we will compute the optical depth at the Lyman continuum limit, $\tau_{912}$, as derived from the two models and we will compare it with previous observations (Schmieder et al. 2003) and with the spectroscopic model of filaments proposed by Heinzel et al. (2003).

\section{Data analysis}

The filaments considered in the present paper are those listed in Table 1, namely the one analysed in Paper I (July 28, 1996 ) and two out of the three analysed in Paper II.

The filament observed on september 17, 1996, called F1 in Paper II, appears not suitable for the present investigation 
Table 1. CDS and SUMER files analysed in this study.

\begin{tabular}{ccc}
\hline \hline Date & CDS files & SUMER files \\
\hline July 17, 1996 & s3843r00 & sum 960728_155424 \\
(FP1) & s3844r00 & sum 960728_162218 \\
& & sum 960728_163608 \\
May 9, 1998 & s11068r00-01 & \\
(F2) & s11069r00-01 & \\
June 20, 1998 & s11491r00 & \\
(F3) & s11491r01 & \\
& s11491r02 & \\
\hline
\end{tabular}

since there are only two data points that could supply information on the HeI absorption with their wavelengths less than one angstrom apart, (MgVII at $367.7 \AA$ and MgIX at 368.0 $\AA$ ), thus making impossible any reliable fit at $\lambda<504 \AA$.

The data analysis of the two filaments considered in Paper II was done using different software than in Paper I, therefore, for the sake of homogeneity we have repeated the analysis of the filament FP1 using the same routines. For this reason the data shown in Paper I may appear different from the present ones, in particular the number of lines analyzed here is larger than for Paper I.

\subsection{Model A}

Let us first assume the isothermal cool prominence model with a PCTR around it. This model assumes a loop-shaped prominence located well above the chromosphere, therefore the background emission of the quiet sun TR below the filament, $I_{\mathrm{bg}}$, affects the observations and must be taken into account.

The ratio between the average intensitiy of a given line observed above the filament, $I_{\mathrm{obs}}$, and that of the same line averaged on a portion of the quiet sun $I_{\mathrm{qs}}$, selected on the same raster, is given by:

$\frac{I_{\mathrm{obs}}}{I_{\mathrm{qs}}}=\frac{I_{\mathrm{bg}}}{I_{\mathrm{qs}}} \mathrm{e}^{-\tau}+\frac{I_{\mathrm{fil}}}{2 I_{\mathrm{qs}}}\left(1+\mathrm{e}^{-\tau}\right)$

where $I_{\text {fil }} / 2$ is the emission of the filament-corona TR at the top and at the bottom of the filament which are assumed equal and $\tau$ is the optical depth due to HI and HeI bound-free absorption.

In the above equation we have neglected the so-called "volume blocking" effect (Heinzel \& Schmieder 2001), namely the lack of emission from the volume occupied by the cool prominence gas. We think that this factor, which is the dominant one in explaining the lower intensity observed in coronal lines above filaments (see Paper I), does not play an important role in Eq. (1), with respect to TR lines. In the framework of model A, only the loop legs are located in the TR while most of the prominence body lies in the corona. Therefore, seen from above, the volume blocking of TR lines affects a very small part of the filament area on which the line intensities are averaged.

Taking the logarithm of the above equation, we get:

$-\ln \left(I_{\mathrm{obs}} / I_{\mathrm{qs}}-f\right)=-\ln (b+f)+\alpha_{\mathrm{H}} x+\alpha_{\mathrm{He}} y$

where $\alpha_{\mathrm{H}}$ and $\alpha_{\mathrm{He}}$ are the $\mathrm{H}$ and $\mathrm{He}$ bound free absorption coefficients (see, for instance Gurzadian 1997), $x$ and $y$ are the neutral $\mathrm{H}$ and He column densities, $b=I_{\mathrm{bg}} / I_{\mathrm{qs}}$ and $f=I_{\mathrm{fil}} / 2 I_{\mathrm{qs}}$.
Table 2. Fit parameters obtained for Model A: $f$ and $b$ are the PCTR and the background intensity, relative to the quiet sun average intensity; $x$ and $y$ are the $\mathrm{HI}$ and HeI column densities in units of $10^{17} \mathrm{~cm}^{-2}$.

\begin{tabular}{ccccccc}
\hline \hline Filament & $f$ & $b$ & $x$ & $y$ & $\mathrm{HeI} / \mathrm{HI}$ & $\chi^{2}$ \\
\hline FP1 & 0. & 0.97 & 4.2 & 0.2 & $4.8(-2)$ & 0.41 \\
& 0.1 & 0.78 & 5.1 & 0.2 & $3.9(-2)$ & 0.65 \\
& 0.2 & 0.60 & 6.5 & 0.3 & $4.0(-2)$ & 1.23 \\
F2 & 0. & 1.14 & 5.6 & 0.5 & $8.4(-2)$ & 0.74 \\
& 0.1 & 0.99 & 7.0 & 0.6 & $8.6(-2)$ & 1.13 \\
& 0.2 & 0.94 & 9.5 & 0.9 & $9.5(-2)$ & 2.1 \\
F3 & 0. & 0.61 & 4.7 & 0.4 & $7.9(-2)$ & 0.22 \\
& 0.1 & 0.49 & 7.2 & 0.6 & $9 .(-2)$ & 0.51 \\
& 0.2 & 1.35 & 19 & 2.5 & $1.3(-1)$ & 3.8 \\
\hline
\end{tabular}

In the two previous papers it was assumed that $I_{\text {fil }} \sim 0$, $\alpha_{\mathrm{He}}=0$ and $\alpha_{\mathrm{H}} \propto \lambda^{3}$. With these assumptions, plotting $-\ln \left(I_{\mathrm{obs}} / I_{\mathrm{qs}}\right)$ vs. $\lambda^{3}, x$ and $b$ were derived from the slope and the intercept of the best fitting line, respectively.

In the system of Eqs. (2) $f$ cannot be determined from the best fit procedure, but it must be given as a free parameter. Then for each value of $f$, which has been varied from 0 to 0.2 in steps of $0.05\left(0 \leq I_{\mathrm{fil}} \leq 0.4 I_{\mathrm{qs}}\right)$, a system of $n_{\text {lines }}$ equations in the three unknowns $x, y$ and $z=\ln (b+f)$ can be solved using a least squares method, provided that $f<I_{\mathrm{obs}} / I_{\mathrm{qs}}$.

The filament FP1 was observed also by SUMER at $\lambda>$ $912 \AA(\tau=0)$, supplying the value of $b+2 f$. Since $\tau \rightarrow 0$ also for $\lambda \rightarrow 0$, the limit of the fitting function for $\lambda \rightarrow 0$ must give the same intensity ratios observed by SUMER: $I_{\mathrm{obs}} / I_{\mathrm{qs}}=$ 1. \pm 0.2 . We have therefore added to the data points a point at $\lambda \simeq 0$ having the same intensity ratio observed by SUMER. The results for the three analysed filaments are shown in Table 2, where we have listed only the parameters obtained for $f=0$, 0.1 and 0.2. All curves are instead plotted in Fig. 1 .

An inspection of Table 2 shows that the best fits (the lowest $\chi^{2}$ values) are always obtained assuming $f=0$. On the other hand, Fig. 1 shows that the fits obtained with different values of $f$ are very similar. The only parameter which shows a strong dependence on $f$ is, of course, the background emission $b$.

\subsection{Model B}

Model B assumes a filament made up of $N$ thin cool threads embedded in the hot coronal plasma, each of which is surrounded by a tube-like TR. With the assumption that the TR emission, $i_{f}$, and the optical depth, $\tau_{f}$, are the same for each thread, the observed intensity ratio $\left(I_{\mathrm{obs}} / I_{\mathrm{qs}}\right)$ is given by (see appendix of Paper II):

$I_{\mathrm{obs}} / I_{\mathrm{qs}}=2 N\left(i_{\mathrm{fil}} / I_{\mathrm{qs}}\right) \cdot g(\tau)+I_{\mathrm{bg}} / I_{\mathrm{qs}} \mathrm{e}^{-\tau}$.

$2 N i_{\text {fil }}=I_{\text {fil }}$ is the total PCTR emission that would be observed, added to $I_{\mathrm{bg}}$, for $\tau=0(\lambda>912 \AA), \tau=N \tau_{f}$ is the total filament opacity and $g(\tau)=\left(1-\mathrm{e}^{-\tau}\right) / \tau$. If we assume that the cool threads are rooted in the chromosphere and that there is no quiet sun TR under the prominence, $I_{\mathrm{bg}}=0$, we may take the logarithm of the above equation. Substituting $-\ln g(\tau)$ with the straight line of equation $-\ln g(\tau)=0.025+0.419 \tau$ which, 

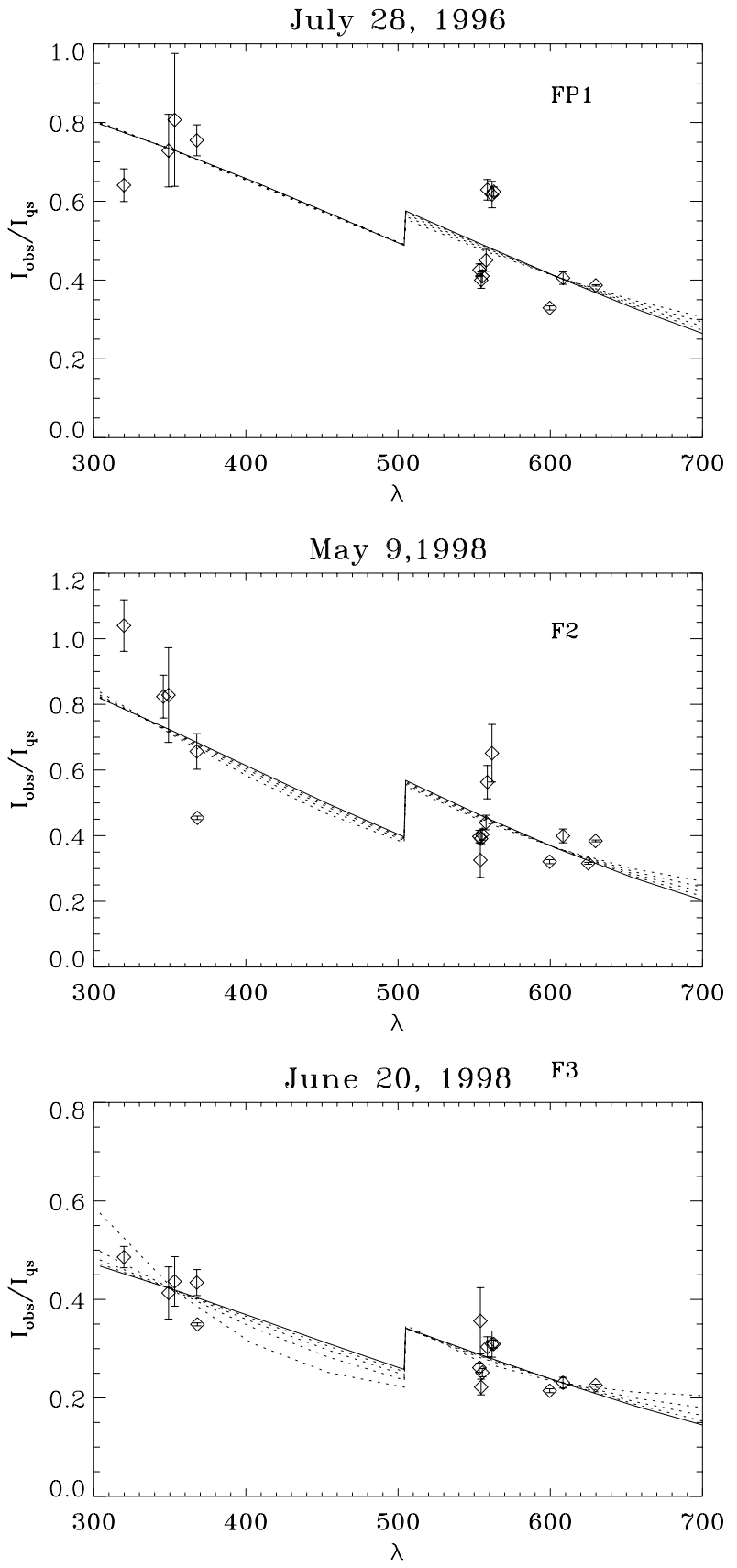

Fig. 1. Fit of the observed average ratios $I_{\mathrm{obs}} / I_{\mathrm{qs}}$ obtained with model A: full line is computed assuming $f=I_{\text {fil }} / 2 I_{\mathrm{qs}}=0$, dotted lines varying $f$ from 0.05 to 0.2 in steps of 0.05 .

in the range $0.3 \leq \tau \leq 2$, fits the curve with an accuracy better than $3 \%$ (see Fig. 2), we get:

$-\ln \frac{I_{\mathrm{obs}}}{I_{\mathrm{qs}}}-0.025=-\ln \frac{I_{\mathrm{fil}}}{I_{\mathrm{qs}}}+0.419\left(\alpha_{\mathrm{H}} x+\alpha_{\mathrm{He}} y\right)$.

This system of $n_{\text {lines }}$ equations in the three unknowns $x, y$ and $z=\ln \left(I_{\mathrm{fil}} / I_{\mathrm{qs}}\right)$ is very symilar to the one previously solved with the total PCTR emission replacing the background emission. The solutions are listed in Table 3 and the data fits are shown in Fig. 3, where the fitting lines are obtained using the exact expression of $g(\tau)$.

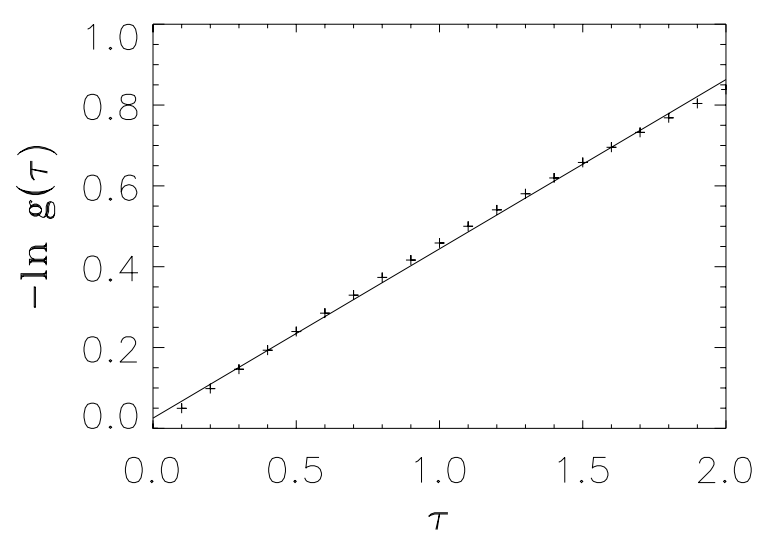

Fig. 2. Linear fit of $-\ln g(\tau)$ appearing in Eq. (3) (crosses) with a straight line of equation $-\ln g(\tau)=0.025+0.419 \tau$.

Table 3. Fit parameters obtained for model B: $x$ and $y$ are the HI and HeI column densities in units of $10^{17} \mathrm{~cm}^{-2}$.

\begin{tabular}{cccccc}
\hline \hline Filament & $I_{\text {fil }} / I_{\mathrm{qs}}$ & $x$ & $y$ & $\mathrm{HeI} / \mathrm{HI}$ & $\chi^{2}$ \\
\hline FP1 & 1.0 & 10.1 & 0.5 & $5(-2)$ & 0.4 \\
F2 & 1.16 & 13.3 & 1.1 & $8.2(-2)$ & 0.7 \\
F3 & 0.62 & 11.1 & 0.9 & $7.9(-2)$ & 0.2 \\
\hline
\end{tabular}

A comparison between the parameters derived with the two models shows that:

a) The values of the PCTR emission, $f=I_{\mathrm{fil}} / I_{\mathrm{qs}}$, are very similar to the values of $b=I_{\mathrm{bg}} / I_{\mathrm{qs}}$, obtained with $f=0$ in model A. We want to recall that, in contrast to Model A, this value of $f$ can be observed only at $\lambda>912 \AA$. At shorter wavelengths the observed filament emission is $I_{\text {fil }} \cdot g(\tau)$. The similarity of $b$ (Model A) and $f$ (Model B) is not surprising since these quantities represent, in both cases, the constant term of the fit, namely the limit of $I_{\mathrm{obs}} / I_{\mathrm{qs}}$ for $\lambda \rightarrow 0$. For $\tau \simeq 0$ we have, with both models:

$I_{\mathrm{obs}}=I_{\mathrm{bg}}+I_{\mathrm{fil}}$.

Therefore, if we put one of the two terms equal to zero, the constant term of the fit supplies the other.

b) The column densities derived with model $\mathrm{B}$ are larger than those of model A by more than a factor of two. This is due to the fact that in model $\mathrm{A}$ the background absorption takes place all along the filament thickness, while in model B each PCTR emission is absorbed only by the higher portion of the filament.

c) The two models supply about the same $\mathrm{HeI} / \mathrm{HI}$ ratios, in spite of the large differences in the column densities. This result make us quite confident in the reliability of the derived $\mathrm{HeI} / \mathrm{HI}$ ratios.

The weakest aspect of model B is the absence of a quiet sun TR under the filament, an assumption adopted for mathematical reasons, to allow derivation of a linear relationship between the observations and the optical depth. In order to check how this assumption could affects our results, we have solved the system of $n_{\text {lines }}$ equations like Eq. (3) in the two unknowns $f$ and $b$ for different values of the HeI abundance and of the H-column density, $x$, finding the following results. Both $f$ and $b$ depend 

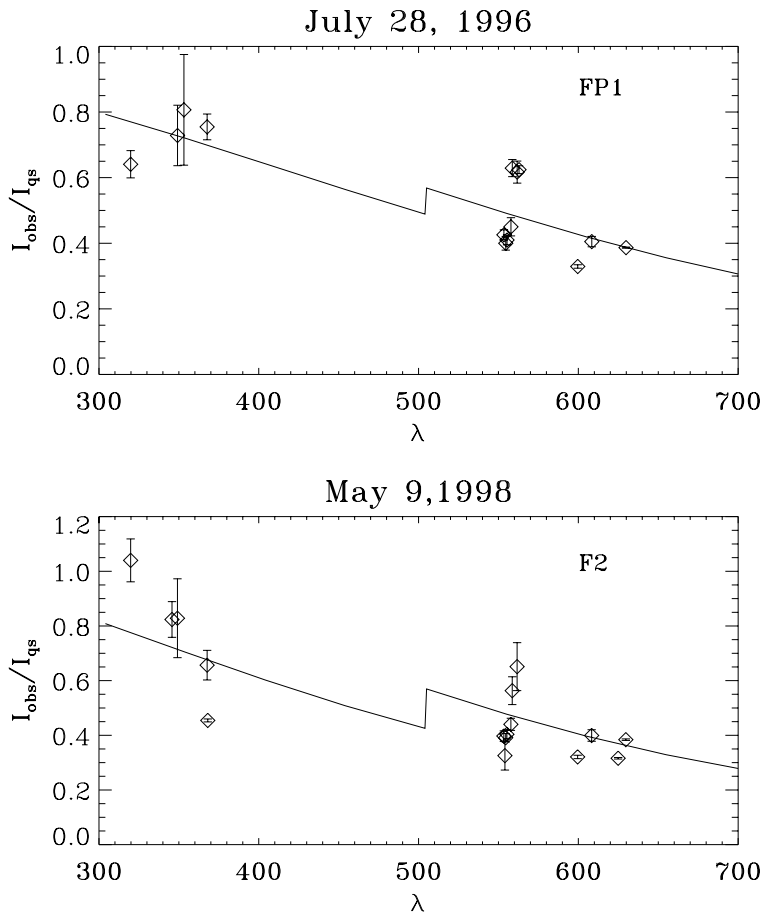

June 20, 1998

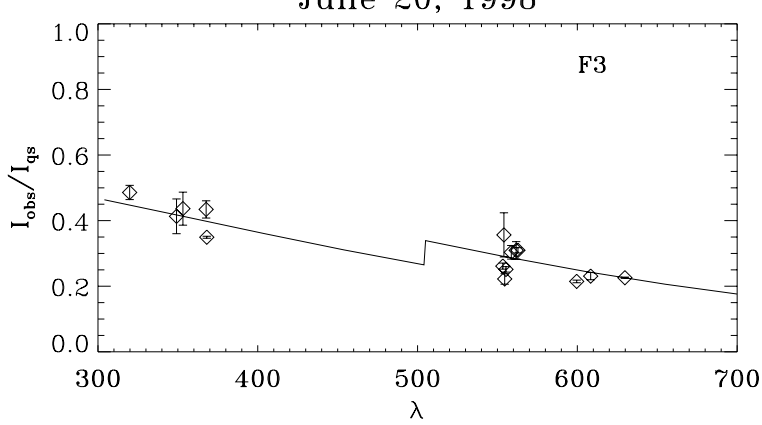

Fig. 3. Fit of the observed average ratios $I_{\mathrm{obs}} / I_{\mathrm{qs}}$ obtained with model B.

very little on the $\mathrm{HeI} / \mathrm{HI}$ ratio, while they strongly depend on $x$. If we take for instance the filament FP1, for which, according to SUMER observations, $f+b=1$, we see that, at the lowest value of $x=4.2 \times 10^{17}$, model B supplies $f=0.05$ and $b=0.95$, in very good agreement with model A. For $x=6 \times 10^{17}$ the two parameters becomes equal, $f=b=0.5$, and, for higher values of $x, f \rightarrow 1$ and $b \rightarrow 0$. Therefore, as already mentioned in point a) above, the assumption that $b=0$ in Model B does not affect the main result of this paper, namely the HeI abundance, though it does affect the derived HI-column density. A further check on this quantity will be presented in the next section.

\section{Determination of $\tau_{912}$}

From the results found in the previous section it appears that the only real differences between the fit parameters derived from the two models are in the column densities, which are much larger in model B than in model A. A choice between the two models can therefore be based only on this quantity.

From the HI column densities, derived with the two models, we have computed the expected optical depth at the Lyman
Table 4. $\tau_{912}$ obtained from the column densities listed in Table 2 (with $f=0$ ) and in Table 3 .

\begin{tabular}{ccc}
\hline \hline Filament & Model A & Model B \\
\hline FP1 & $2.6 \pm 0.6$ & $6.4 \pm 1.5$ \\
F2 & $3.5 \pm 1.5$ & $8.4 \pm 3.7$ \\
F3 & $3 . \pm 1$. & $7 . \pm 2.4$ \\
\hline
\end{tabular}

continuum limit, $\tau_{912}=6.3 \times 10^{-18} x$, which can be compared with previous observations of different filaments. The values of $\tau_{912}$ obtained with the two models are listed in Table 4.

We see that the average value of $\tau_{912}=5.3$, found by Schmieder et al. (2003), falls in the ranges of $\tau_{912}$ derived from model B. Moreover only these latter values of $\tau_{912}$ appear to satisfy the requirements of the spectroscopic model of Heinzel et al. (2003). According to these authors, realistic values of the height of the prominence lower and upper boundaries $\left(h_{3}\right.$ and $h_{4}$ ) are consistent with $3.8 \leq \tau_{912} \leq 8.4$.

\section{Discussion and conclusions}

In this paper we have analysed TR lines observed in three different filaments by the CDS instrument onboard the SoHO satellite. The main purpose of this research was to check if the $\mathrm{HeI} / \mathrm{HI}$ ratio in prominences varies from one feature to the other as suggested in Paper II. The determination done in the present paper shows that in all filaments the $\mathrm{HeI} / \mathrm{HI}$ ratio varies from 0.05 to 0.08 , independently of the assumed model.

In the assumption of thermodynamic equilibrium, the $\mathrm{HeI} / \mathrm{HI}$ ratio cannot be lower than the He abundance, [He], since this would imply that the first ionization potential of HeI is lower than that of HI. Let us assume that the He abundance in prominences is the same as in the low corona: if we take, according to Gabriel et al. (1995), $[\mathrm{He}]=0.08$, this means that in solar prominences both $\mathrm{H}$ and $\mathrm{He}$ are neutral $\left(i=j_{1}=j_{2}=0\right.$ in Anzer \& Heinzel 2005, notations), while assuming the Laming $\&$ Feldman (2001) low corona abundance $[\mathrm{He}]=0.05$, we derive that in $\mathrm{F} 2$ and $\mathrm{F} 3 i>j_{1}$ while in $\mathrm{FP} 1 i=j_{1}=0$.

This would therefore imply that all prominences have about the same temperature, $T<10^{4}$, if $[\mathrm{He}]=0.08$ is assumed, while $[\mathrm{He}]=0.05$ could suggest that $\mathrm{F} 2$ and $\mathrm{F} 3$ have a temperature higher $\left(T \sim 1.4 \times 10^{4} \mathrm{~K}\right)$ than FP1 $\left(T<10^{4}\right)$. However, according to Anzer \& Heinzel (2005), the ionization rates of both $\mathrm{H}$ and $\mathrm{He}$ in prominences are strongly affected by other factors besides the temperature, therefore we cannot make any safe speculation on the prominence temperature.

Another interesting result presented in this paper is the determination of optical depth of the filaments at the Ly-C limit, $\tau_{912}$ using the HI column densities as derived from the two models, A and B. The comparison of these results with previous determination (Schmieder et al. 2003) and mostly with the requirements of the spectroscopic model of Heinzel et al. (2003) indicates that only model B can supply values in agreement with the above findings.

The measurement of the volume blocking of coronal lines in FP1, given in Paper I, was also unambiguously in favor of model B. As already mentioned, the volume blocking, which 
we argued not to be very important for TR lines, becomes the most important factor for the reduced intensity observed above filaments in coronal lines. For these lines the Lyman absorption is not very important since it affects only the radiation coming from the coronal slab under the filament. The missing volume of hot coronal plasma is instead very large. Thus it is much larger in model $\mathrm{A}$, where it is equal to the total prominence volume, than in model $\mathrm{B}$, where it reduces to the volume of the cool threads plus that of the tube-like PCTR. It appears therefore straightforward to discriminate between the two models A and B from coronal lines. Moreover we suggest that the "weakest point" of model B mentioned above, namely the absence of the quiet sun TR under the filament, does not affect the coronal lines. We propose to extend the calculation of the volume blocking using coronal lines to the other three filaments in a forthcoming paper.

Acknowledgements. SOHO is a project of international cooperation between ESA and NASA. The author is indebted to G. Del Zanna for providing her with his routines for CDS data reduction and to
C. Chiuderi and S. Parenti for helpful discussion and suggestion. She wishes moreover to thank the unknown referee for the useful comments and remarks to the paper.

\section{References}

Anzer, U., \& Heinzel, P. 2005, ApJ, 622, 721

Chiuderi Drago, F., Engvold, O., \& Jensen, E. 1992, Sol. Phys. 139, 47

Chiuderi Drago, F., Alissandrakis, C. E., Bastian, et al. 2001, Sol. Phys. 199, 115 (Paper I)

Del Zanna, G., Chiuderi Drago, F., \& Parenti, S. 2004, A\&A, 420, 307 (Paper II)

Gabriel, A. H., Culhane, J. L., Patchett, B. E., et al. 1995, Adv. Sp. Res., 15, 63

Gurzadian, G. H. 1997, Physics and Dynamics of Planetary Nebulae (Springer-Verlag), 48

Heinzel, P., Schmieder, B., \& Tziotziou, K. 2001, ApJ, 561, L223

Heinzel, P., Anzer, U., \& Schmieder, B. 2003, Sol. Phys., 216, 159

Laming, J., M., \& Feldman, U. 2001, ApJ, 546, 552

Schmieder, B., Tziotziou, K., \& Heinzel, P. 2003, A\&A, 401, 361 